\title{
Stress Signal ULBP4, an NKG2D Ligand, Is Upregulated in Multiple Sclerosis and Shapes $\mathrm{CD8}^{+}$ T-Cell Behaviors
}

Ana Carmena Moratalla, MSc, Yves Carpentier Solorio, BSc, Florent Lemaitre, MSc, Negar Farzam-kia, BSc, Annie Levert, DEC, Stephanie E.J. Zandee, PhD, Boaz Lahav, BSc, Jean Victor Guimond, MD, Elie Haddad, MD, PhD, Marc Girard, MD, Pierre Duquette, MD, Catherine Larochelle, MD, PhD, Alexandre Prat, MD, PhD, and Nathalie Arbour, PhD

Neurol Neuroimmunol Neuroinflamm 2022;9:e1119. doi:10.1212/NXI.0000000000001119

\section{Abstract}

\section{Background and Objectives}

We posit the involvement of the natural killer group 2D (NKG2D) pathway in multiple sclerosis (MS) pathology via the presence of specific NKG2D ligands (NKG2DLs). We aim to evaluate the expression of NKG2DLs in the CNS and CSF of patients with MS and to identify cellular stressors inducing the expression of UL16-binding protein 4 (ULBP4), the only detectable NKG2DL. Finally, we evaluate the impact of ULBP4 on functions such as cytokine production and motility by $\mathrm{CD}^{+} \mathrm{T}$ lymphocytes, a subset largely expressing NKG2D, the cognate receptor.

\section{Methods}

Human postmortem brain samples and CSF from patients with MS and controls were used to evaluate NKG2DL expression. In vitro assays using primary cultures of human astrocytes and neurons were performed to identify stressors inducing ULBP4 expression. Human CD8 ${ }^{+}$ T lymphocytes from MS donors and age/sex-matched healthy controls were isolated to evaluate the functional impact of soluble ULBP4.

\section{Results}

We detected mRNA coding for the 8 identified human NKG2DLs in brain samples from patients with MS and controls, but only ULBP4 protein expression was detectable by Western blot. ULBP4 levels were greater in patients with MS, particularly in active and chronic active lesions and normal-appearing white matter, compared with normal-appearing gray matter from MS donors and white and gray matter from controls. Soluble ULBP4 was also detected in CSF of patients with MS and controls, but a smaller shed/soluble form of $25 \mathrm{kDa}$ was significantly elevated in CSF from female patients with MS compared with controls and male patients with MS. Our data indicate that soluble ULBP4 affects various functions of $\mathrm{CD} 8^{+} \mathrm{T}$ lymphocytes. First, it enhanced the production of the proinflammatory cytokines GM-CSF and interferon- $\gamma$ (IFN $\gamma$ ). Second, it increased $\mathrm{CD}^{+} \mathrm{T}$ lymphocyte motility and favored a kinapse-like behavior when cultured in the presence of human astrocytes. $\mathrm{CD} 8^{+} \mathrm{T}$ lymphocytes from patients with MS were especially altered by the presence of soluble ULBP4 compared with healthy controls.

\section{Discussion}

Our study provides new evidence for the involvement of NKG2D and its ligand ULBP4 in MS pathology. Our results point to ULBP4 as a viable target to specifically block 1 component of the NKG2D pathway without altering immune surveillance involving other NKG2DL.
Correspondence

Dr. Arbour

nathalie.arbour@umontreal.ca
MORE ONLINE

$\checkmark$ Videos

From the Department of Neurosciences (A.C.M., Y.C.S., F.L., N.F-k., A.L., S.E.J.Z., M.G., P.D., C.L., A.P., N.A.), Université de Montréal and Centre de Recherche du CHUM (CRCHUM) Montreal; MS-CHUM Clinic (B.L., M.G., P.D., C.L., A.P.); CLSC des Faubourgs (J.V.G.), CIUSSS du Centre-Sud-de-I'lle-de-Montréal; and Department of Microbiology, Infectious Diseases, and Immunology and Department of Pediatrics (E.H.), Universite de Montréal, Centre de Recherche du Centre Hospitalier Universitaire Sainte-Justine (CHU Sainte-Justine), Montreal, Quebec, Canada.

Go to Neurology.org/NN for full disclosures. Funding information is provided at the end of the article.

The Article Processing Charge was funded by the authors.

This is an open access article distributed under the terms of the Creative Commons Attribution-NonCommercial-NoDerivatives License 4.0 (CC BY-NC-ND), which permits downloading and sharing the work provided it is properly cited. The work cannot be changed in any way or used commercially without permission from the journal. 


\section{Glossary}

BBB = blood-brain barrier; $\mathbf{C A}=$ chronic active; $\mathbf{E A E}=$ experimental autoimmune encephalomyelitis; $\mathbf{E R}=$ endoplasmic reticulum; GBM = glioblastoma multiforme; GFAP = glial fibrillary acidic protein; GM-CSF = granulocyte-macrophage colonystimulating factor; IFN $\gamma=$ interferon- $\gamma ;$ MIC = major histocompatibility complex class I chain-related protein; MICA = major histocompatibility complex class I chain-related protein A; MICB = major histocompatibility complex class I chain-related protein B; MS = multiple sclerosis; MULT1 = murine UL16-binding proteinlike transcript 1; NAGM = normal-appearing gray matter; NAWM = normal-appearing white matter; NK = natural killer; NKG2D = natural killer group 2D; OND = other neurologic disorder; PPMS = primary progressive MS; rhULBP4 = recombinant human ULBP4; RRMS = relapsing-remitting MS; SPMS = secondary progressive MS; TNF = tumor necrosis factor; ULBP4 = UL16-binding protein 4.

Multiple sclerosis (MS) is a chronic inflammatory disease of the CNS. The neuropathologic hallmarks include demyelinating areas, neuronal/axonal loss, and gliosis. ${ }^{1}$ Reactive astrocytes, defined as those "undergoing morphological, molecular, and functional changes in response to pathologic situations," ${ }^{2}$ are commonly present in MS lesions. The key roles of astrocytes in shaping local inflammatory responses are increasingly recognized; indeed, these glial cells interact with other neural cells as well as infiltrating leukocytes. ${ }^{3}$ Notably, CNS-infiltrating immune cells are associated with active demyelination and neurodegeneration in MS. ${ }^{1}$ Among infiltrating lymphocytes, $\mathrm{CD} 8^{+}$ $\mathrm{T}$ lymphocytes are very abundant, although their contribution to MS pathobiology is incompletely resolved. ${ }^{1}$

Natural killer group 2D (NKG2D) is a (co)activating receptor expressed by immune effector cells including natual killer (NK) cells, $\mathrm{CD}^{+} \mathrm{T}$ lymphocytes, and subsets of $\mathrm{CD} 4^{+} \mathrm{T}$ lymphocytes. NKG2D acts on numerous immune functions; for example, it provides costimulation to $\mathrm{T}$ lymphocytes increasing cytotoxicity, chemokine responsiveness, and inflammatory cytokine production. ${ }^{4}$ NKG2D-expressing $T$ lymphocytes are enriched in sites targeted by chronic inflammatory diseases and are associated with the production of inflammatory mediators such as in active skin lesions of patients with vitiligo, intestinal epithelium of patients with active celiac disease, and gut lamina propria of patients with Crohn disease. ${ }^{5,6}$ Results from others and our group pinpoint the NKG2D pathway as a key player in MS pathology, especially through T lymphocytemediated mechanisms. In MS brain lesions, all infiltrating $\mathrm{CD}^{+} \mathrm{T}$ lymphocytes express NKG2D, and the number of $\mathrm{NKG}_{2} \mathrm{D}^{+} \mathrm{CD} 4^{+} \mathrm{T}$ lymphocytes is elevated compared with non-MS controls. ${ }^{7} \mathrm{NKG}_{2} \mathrm{D}^{+} \mathrm{CD} 4^{+} \mathrm{T}$ lymphocytes are more abundant in the blood of patients with MS during relapse, and these $\mathrm{T}$ lymphocytes have an encephalitogenic profile, with enhanced cytolytic and migratory capacities. ${ }^{7}$ In addition, we showed that passive experimental autoimmune encephalomyelitis (EAE) is less severe in NKG2D-deficient recipient mice than their wild-type counterparts. ${ }^{8}$ Moreover, conditional deletion of NKG2D in T lymphocytes reduced active EAE disease severity compared with wild-type counterparts. ${ }^{9}$

NKG2D can recognize multiple ligands (referred as NKG2DLs) grouped in humans into the major histocompatibility complex class I chain-related protein (MIC) (MICA and MICB) and
UL16-binding protein (ULBP 1-6) families. ${ }^{10}$ Under normal physiologic conditions, these ligands are expressed at low or undetectable levels but are induced by cellular stress, serving to alert the immune system. ${ }^{10}$ Accumulating evidence supports the notion that distinct cell types upregulate specific NKG2DLs in response to different stresses, including DNA damage, inflammation, oxidative stress, and endoplasmic reticulum (ER) stress. ${ }^{10,11}$ The surface protein expression of each NKG2DL is finely regulated by multiple mechanisms at the transcriptional, translational, and posttranslational levels including shedding of soluble forms. ${ }^{10,11}$ Therefore, NKG2DL expression is cell type and environment specific, and such diversity of ligand expression could facilitate the targeting of precise elements of the NKG2D pathway depending on disease context. ${ }^{10,11}$

Several cellular stressors (i.e., inflammation and oxidative stress) are present in MS brains ${ }^{1}$; whether they trigger NKG2DLs in specific human CNS cell types is unresolved. Elevated levels of soluble NKG2DLs in biological fluids of other inflammatory diseases are linked to enhanced autoimmune T lymphocyte activation. ${ }^{12}$ Soluble MICA/B is detected in the serum of patients with $\mathrm{MS},{ }^{13}$ but there are no data available for CSF. We have previously detected $\mathrm{MICA} / \mathrm{B}^{+}$oligodendrocytes in MS brain tissue; we also observed $\mathrm{CD} 8^{+}$ $\mathrm{T}$ lymphocytes in close proximity to these $\mathrm{MICA} / \mathrm{B}^{+}$cells. Therefore, our results support the possibility of in vivo contact between $\mathrm{T}$ lymphocytes and NKG2DL ${ }^{+} \mathrm{CNS}$ cells in MS. ${ }^{14}$ Whether other neural cells in patients with MS express specific NKG2DLs and therefore may be recognized by NKG2Dexpressing $\mathrm{T}$ lymphocytes has not been determined.

Here, we investigate whether specific NKG2DLs could be involved in MS pathobiology. We found higher levels of ULBP4 in the brain of patients with MS compared with controls and identified astrocytes as the predominant cell type expressing this ligand. In addition, we showed elevated levels of soluble ULBP4 in the CSF of female patients with MS. Finally, we demonstrate that soluble ULBP4 enhances the secretion of inflammatory cytokines by $\mathrm{CD} 8^{+} \mathrm{T}$ lymphocytes. Moreover, the addition of soluble ULBP4 alters the motility and behavior of $\mathrm{CD} 8^{+} \mathrm{T}$ lymphocytes cocultured with human astrocytes. Our results support the involvement of ULBP4 in the neuroinflammatory context of MS pathology. 


\section{Methods}

\section{Ethics}

Written informed consent was obtained from patients with MS and controls in accordance with the local ethical committee, and studies were approved by the Centre Hospitalier de l'Université de Montréal ethical board (BH 07.001, HD 07.002). Patients were recruited from the CHUM MS Clinic and clinically classified as relapsing-remitting MS (RRMS), primary progressive MS (PPMS), or secondary progressive MS (SPMS) according to the revised $2017 \mathrm{McDonald}$ criteria ${ }^{15}$ by a highly trained MS neurologist (P.D., M.G., C.L., or A.P.). CSF was obtained from patients undergoing lumbar puncture for diagnostic purpose; these samples included untreated patients confirmed as MS or affected by other neurologic disorder (OND). OND included patients diagnosed with migraine, chronic fatigue, epilepsy, or postoperative complications. The number of patients and controls for each assay is indicated in figure legends.

\section{Standard Protocol Approvals, Registrations, and Patient Consents}

Human fetal brain tissue (16-23 weeks old) was obtained on obtaining written informed consent (ethical committee of CHU Sainte-Justine, CER\#2126; University of Washington Birth Defects Research Laboratory Seattle, WA, STUDY00000380).
These studies were approved by the CHUM ethics boards (BH07.001, HD07.002).

\section{Statistical Analysis}

Data analysis was performed using GraphPad Prism 9.2 software (San Diego, CA). When data passed the Shapiro-Wilk or the D'Agostino-Pearson normality test, the paired $t$ test or 1-way analysis of variance was performed, and data are presented as mean \pm SEM. When data did not pass the normality test, the Wilcoxon test, Friedman test, or Kruskal-Wallis test was used, and data are presented as mean. Values were considered statistically significant when probability $(p)$ values were as follows: $p \leq 0.05\left(^{*}\right), p \leq 0.01\left(^{* *}\right), p \leq 0.001\left(^{* * *}\right)$, or $p \leq 0.0001\left(^{* * * *}\right)$.

\section{Data Availability}

Data not provided in the article because of space limitations can be shared at the request of other investigators for the purpose of replicating procedures and results.

\section{Results}

\section{ULBP4 Is Elevated in Brain Lesions and Normal- Appearing White Matter From Patients With MS}

To assess whether NKG2DLs are expressed in human brains, we extracted RNA from snap-frozen brain tissues from patients

Figure 1 ULBP4 Expression Is Elevated in Brain Lesions From Patients With MS

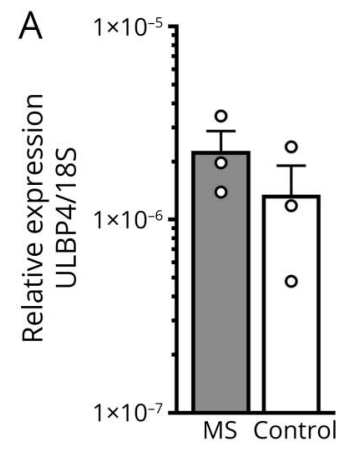

C.a

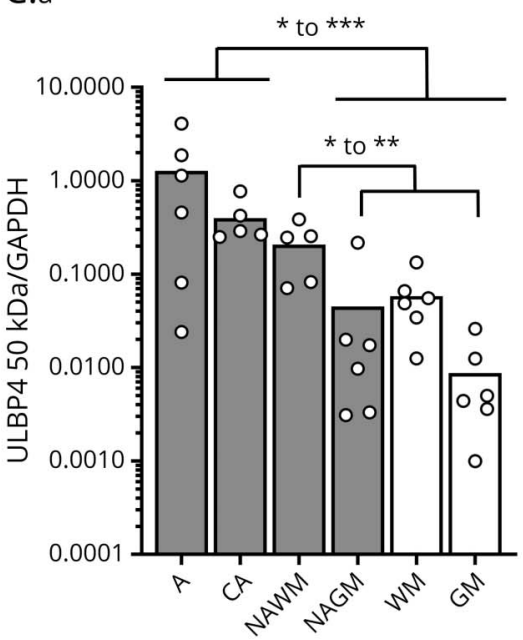

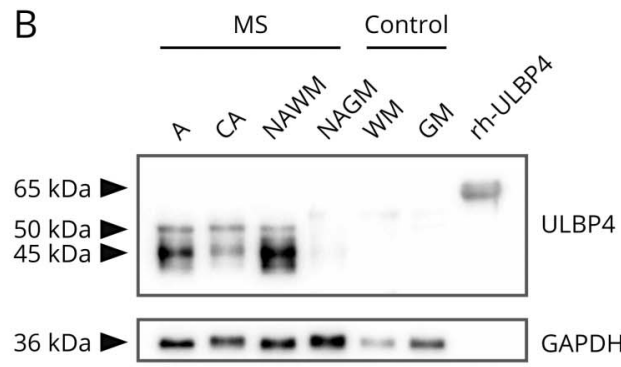

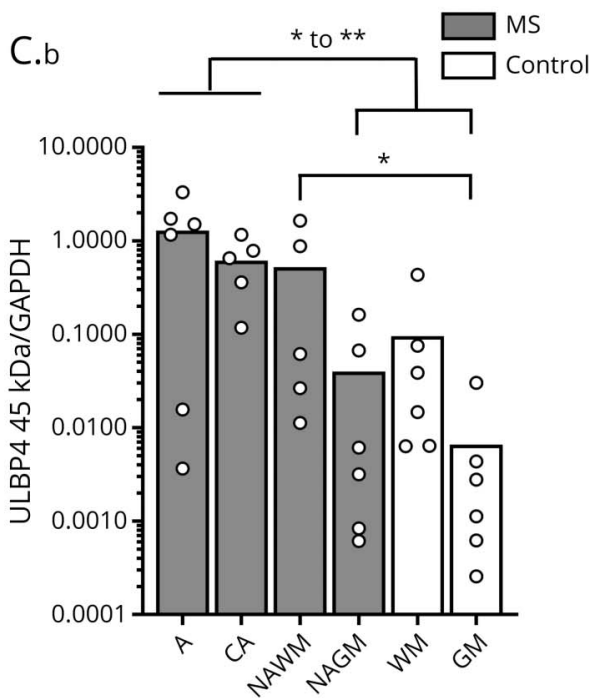

(A) Relative mRNA expression of ULBP4 in postmortem brain samples from untreated patients with MS and controls (epilepsy) (Ctl) expressed as 2$\Delta \mathrm{Ct}$ compared with $18 \mathrm{~S}$. Each dot represents 1 donor $(n=3$ per group). Data are shown as mean + SEM. (B and C) Western blot analysis of ULBP4 in postmortem brain lysates from patients with MS and controls (Ctl). MS samples were characterized as active (A) MS lesion, chronic active (CA) MS lesion, NAWM, and NAGM. Control samples were dissected from either white or gray matter (WM/GM). (B) Representative Western blot showing ULBP4 and GAPDH detection in samples from 1 MS donor and 1 control as well as the positive control rhULBP4. (C) Quantification of $50 \mathrm{kDa}$ (C.a) and 45 $\mathrm{kDa}$ (C.b) ULBP4 bands relative to GAPDH levels. Each dot represents 1 donor. Data are shown as mean for 5-6 patients with MS (5 untreated SPMS and 1 fingolimod-treated transitional RRMS-SPMS) and 6 controls. Comparison between different groups is indicated with lines: Kruskal-Wallis test and uncorrected Dunn test; ${ }^{*} p<0.05$, $* * p<0.01$, and $* * * p<0.001$. CA $=$ chronic active; GAPDH = glyceraldehyde 3-phosphate dehydrogenase; $\mathrm{MS}=$ multiple sclerosis; NAGM = normal-appearing gray matter; NAWM = normal-appearing white matter; rhULBP4 = recombinant human ULBP4; RRMS = relapsing-remitting MS; SPMS = secondary progressive MS; ULBP4 = UL16-binding protein 4. 
with MS and controls and performed real-time quantitative reverse transcription-PCR (eMethods, links.lww.com/NXI/ A669). mRNA coding for the 8 identified human NKG2DLs (ULBP 1-6 and MICA, B) was detected in all brain samples tested (Figure 1A and eFigure 1A). Because these ligands are tightly regulated at multiple levels shaping their protein expression, ${ }^{11}$ we evaluated the protein expression of NKG2DLs by Western blot (eMethods). Although ULBP4 was detected in human brain lysates, levels of other NKG2DLs (ULBP1, ULBP2/5/6, ULBP3, and MICA/B) were below detection in these samples (Figure 1, B and $\mathrm{C}$ and eFigure 1B). To characterize the expression of ULBP4 in the brain, active (A) and chronic active (CA) MS lesions, normal-appearing white matter (NAWM), and normal-appearing gray matter (NAGM) from patients with MS and white and gray matter from controls were compared. Two main bands were detected at approximately 45 and $50 \mathrm{kDa}$, corresponding to ULBP4 (Figure 1B). To validate the specificity of ULBP4 detection, recombinant human ULBP4 (rhULBP4), which has an additional tail, was included as a positive control and was detected at the expected size $(65 \mathrm{kDa})$ reported by the vendor. We also confirmed the specificity of ULBP4 detection as preabsorption of the antiULBP4 antibody with the rhULBP4 abolished ULBP4 detection from protein lysates (data not shown). Quantification of ULBP4 relative to the endogenous control (glyceraldehyde 3-phosphate dehydrogenase) indicated that the $50-\mathrm{kDa}$
ULBP4 band was significantly elevated in both types of MS lesions (A and CA) compared with NAGM from patients with MS and both white and gray matters from controls (Figure 1, B and C). Relative amounts of the ULBP4 $50 \mathrm{kDa}$ band were also significantly greater in NAWM from patients with MS than in gray matter from normal-appearing MS brain and controls. The 45-kDa ULBP4 band was significantly more abundant in active and CA MS lesions compared with NAGM from patients with MS and gray matter from controls. Taken together, our results indicate that the protein levels of ULBP4 are elevated in postmortem brain tissue from patients with MS, especially in MS lesions compared with controls.

\section{ULBP4 Is Mainly Expressed by Astrocytes in MS Brains}

To characterize the ULBP4 expression in postmortem brain tissues, we performed immunostaining on paraffin-embedded brain sections from 4 patients with $\mathrm{MS}$ and 4 controls (Figure 2, eMethods, links.lww.com/NXI/A669). ULBP4 expression was elevated in MS brain sections compared with controls, especially in MS lesions compared with NAGM (Figure 2A). Despite the low abundance of ULBP4 detected by Western blot in controls (Figure 1B), ULBP4-expressing cells were observed in control samples by immunohistostaining (Figure 2A). To identify the cell types with detectable ULBP4, cellular markers for astrocytes (glial fibrillary acidic

Figure 2 ULBP4 Is Mainly Expressed by Astrocytes in Brain Tissue From Patients With MS and Controls

A
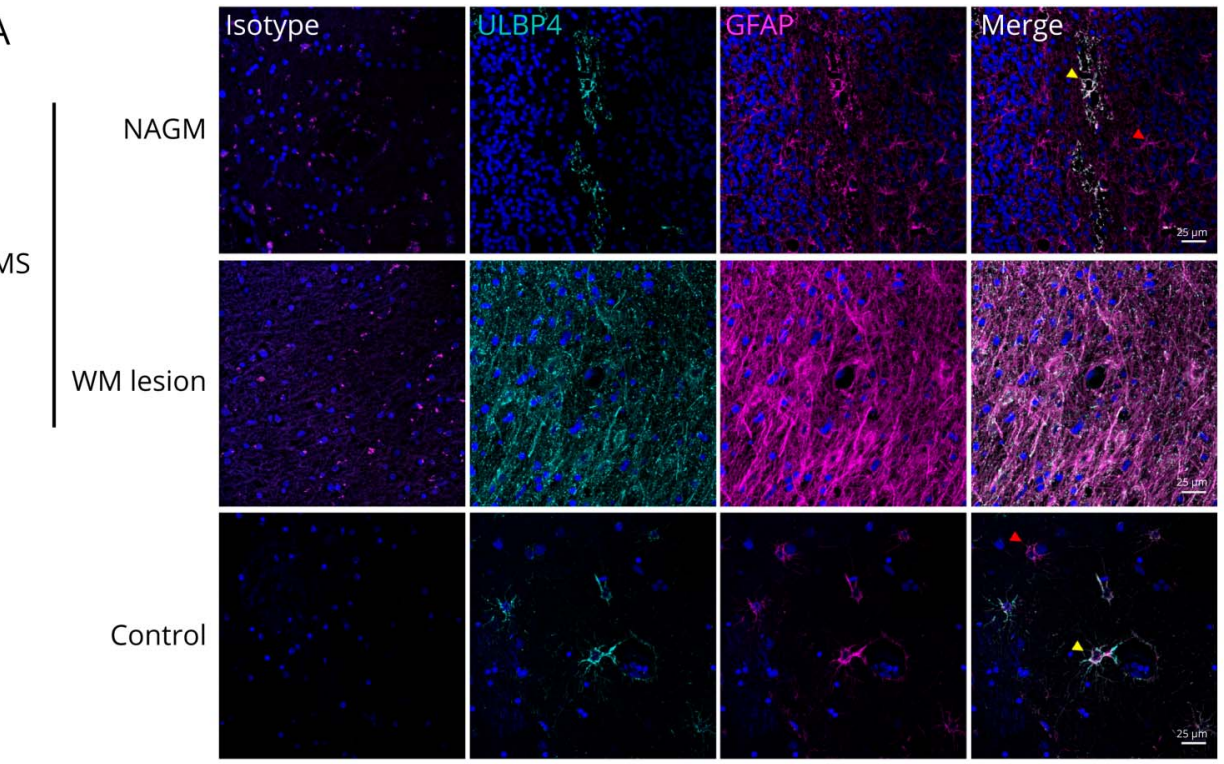

B
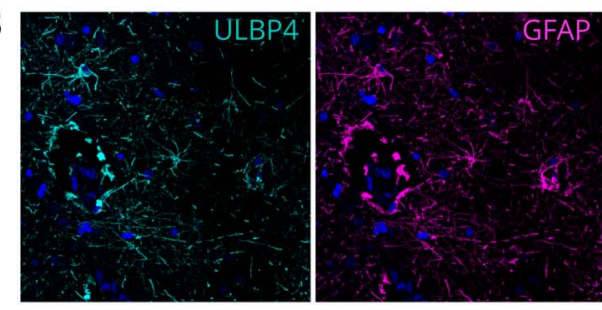

Merge

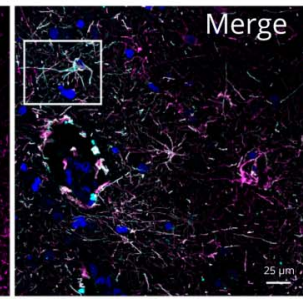

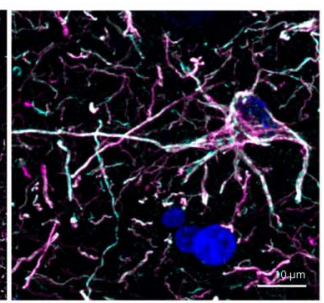

(A) Representative images for the costaining of ULBP4 (cyan) and GFAP (magenta) in paraffin-embedded sections from untreated MS donors (NAGM and WM lesion) and controls (no brain related disease); $n=4$ for each group. Nuclei were stained with 4,6-diamidino-2-phenylindole (blue). Corresponding isotype controls are shown. Yellow arrows indicate $\mathrm{ULBP}^{+} \mathrm{GFAP}^{+}$ cells, and red arrows indicate ULBP4negative $\mathrm{GFAP}^{+}$cells. Scale bars = $25 \mu \mathrm{M}$. (B) Representative enlarged image showing colocalization of GFAP and ULBP4 in the brain section. Scale bars $=10 \mu \mathrm{M}$. GFAP = glial fibrillary acidic protein; MS = multiple sclerosis; NAGM = normal-appearing gray matter; WM = white mater; ULBP4 = UL16binding protein 4. 
protein $[\mathrm{GFAP}])$ and neurons (microtubule associated protein 2 [MAP2]) were used (eMethods). We noted an important colocalization of ULBP4 with GFAP (yellow arrows), but not $\mathrm{MAP2}^{+}$cells, confirming that astrocytes, and not neurons, are the predominant cells expressing ULBP4 (Figure 2B, eFigure 2). A subset of $\mathrm{GFAP}^{+}$cells that did not express ULBP4 (red arrows) was also noted. In summary, our results identify astrocytes as the predominant cell type expressing ULBP4 in the brain of patients with MS.

\section{Cellular Stress Increases ULBP4 Expression by Human Astrocytes}

Multiple cellular stressors can increase NKG2DLs in a cell type specific fashion. ${ }^{10}$ Moreover, brain cells, including astrocytes,

Figure 3 Several Types of Cellular Stress Increase the Proportion of ULBP4-Expressing Astrocytes
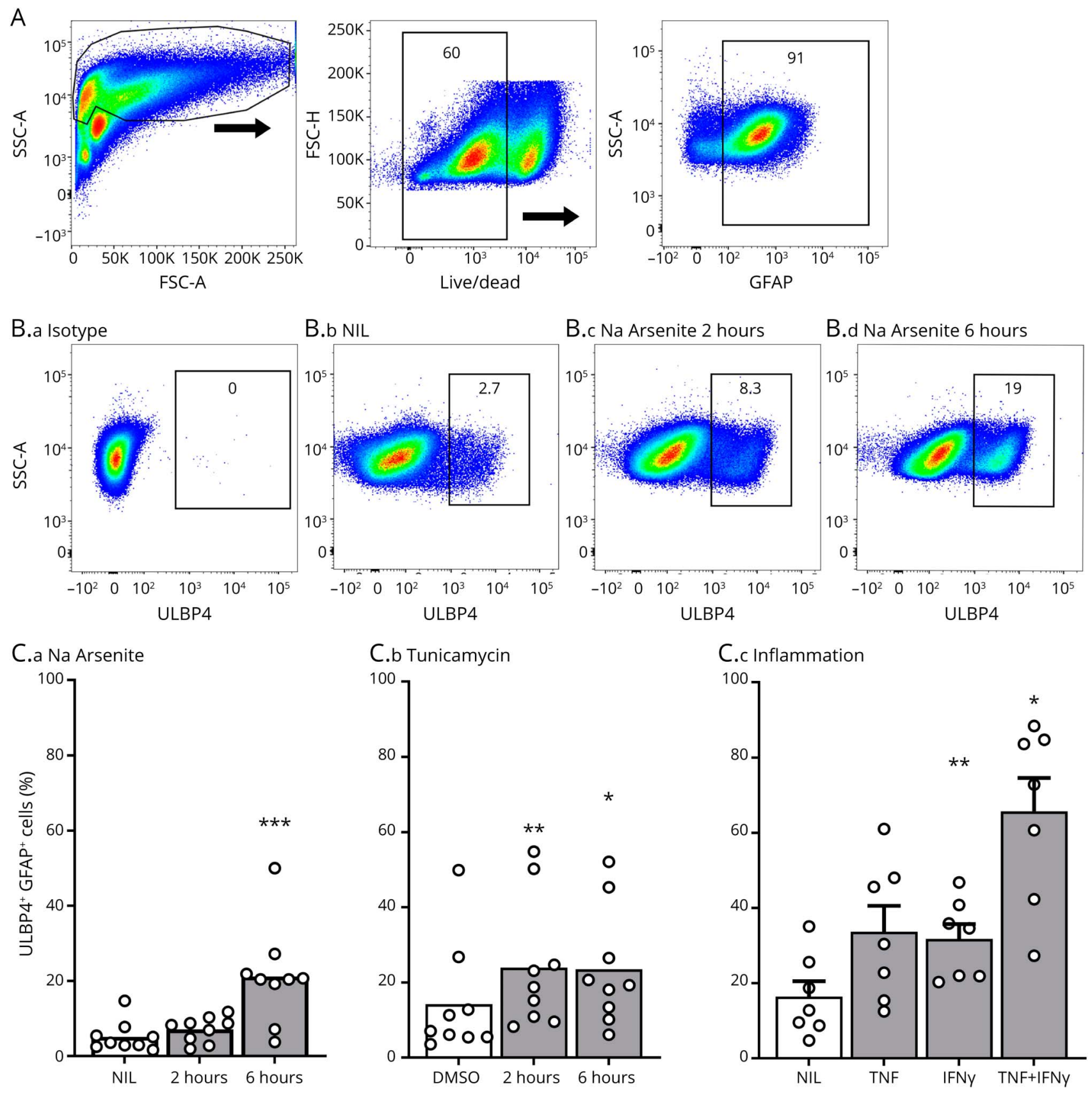

(A-C) Flow cytometry analysis of ULBP4 expression by human astrocytes. (A) Gating strategy from 1 representative donor. Cell debris, doublets, and dead cells were excluded, and GFAP+ cells were selected for analysis. (B) Representative dot plots showing isotype control (B.a) or ULBP4 detection on living GFAP+ gated cells. Astrocytes were either kept under normal culture conditions (NIL) (B.b) or exposed to sodium arsenite (Na Arsenite) for 2 (B.c) and 6 hours (B.d). Percentage of ULBP4+ cells is indicated. (C) Quantification of ULBP4 expression on GFAP+ cells after exposure to sodium arsenite (C.a), tunicamycin (C.b), or proinflammatory cytokines (C.c). Each dot represents 1 donor. Data are shown as mean or mean +SEM, $n=7-9$. Friedman test and Dunn multiple comparison test comparing NIL vs

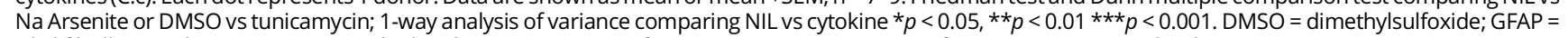
Glial fibrillary acidic protein; MS = multiple sclerosis; IFNy = interferon-y; TNF = tumor necrosis factor; ULBP4 = UL16-binding protein 4. 
exhibit markers of ER and oxidative stress in MS lesions, ${ }^{1,16}$ and several proinflammatory cytokines, including tumor necrosis factor (TNF) and interferon- $\gamma(\mathrm{IFN} \gamma)$, are elevated in MS brain tissues. ${ }^{17}$ To identify cellular stressors that upregulate ULBP4 expression in astrocytes, the main source of ULBP4 in brain tissues, we used our wellestablished primary cultures of human astrocytes and assessed ULBP4 expression by flow cytometry (Figure 3, eMethods, links.lww.com/NXI/A669). We exposed astrocytes to 3 types of cellular stress that are relevant in the context of MS: inflammation, ER stress and oxidative stress, as modeled by $\mathrm{TNF} / \mathrm{IFN} \gamma$, tunicamycin, and sodium arsenite, respectively. Concentrations and exposure time of stressors were validated so as to avoid significant cell death. Following exposure to these stressors, a significantly increased proportion of astrocytes expressed ULBP4, with the TNF/IFN $\gamma$ combination inducing the strongest increase (Figure 3, B and C). In contrast, these cellular stressors did not significantly alter the low ULBP4 expression by human neurons isolated from the same CNS tissue as astrocytes (eFigure 2, B-D). Thus, these results demonstrate that human astrocytes increase ULBP4 expression in response to inflammation, ER, and oxidative stress.

Figure 4 Soluble ULBP4 Is Elevated in CSF From Female Patients With MS and Enhances Proinflammatory Properties of CD8 ${ }^{+}$T Lymphocytes

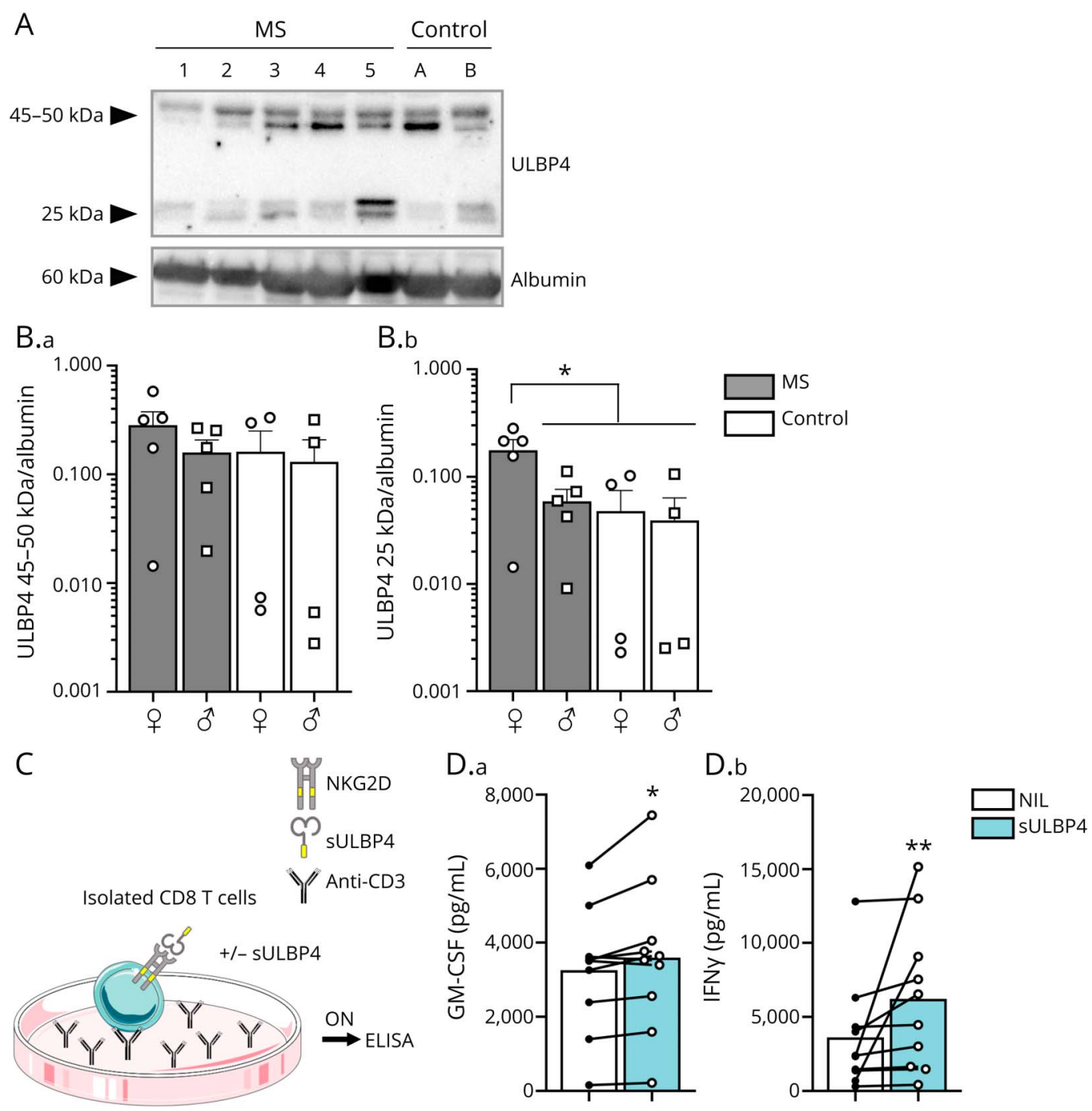

(A and B) Western blot analysis of ULBP4 and albumin levels in CSF from patients with MS and controls (CtI). (A) Representative Western blot including 5 untreated MS donors (1-5) and 2 controls (A and B) illustrating expression of ULBP4 and albumin. (B) Quantification of 45-50 kDa (B.a) and 25 kDa (B.b) bands of ULBP4 relative to albumin according to sex (o: female; $\sigma^{\prime}$ : male) and disease (MS vs control). Each dot represents 1 donor. Mean \pm SEM, $n=5$ female untreated MS, $n=5$ male untreated MS, $n=4$ female controls, and $n=4$ male controls. (C and D) Production of GM-CSF and IFNy by CD8 ${ }^{+} T$ lymphocytes stimulated with or without rhULBP4. (C) Diagram illustrating culture conditions: isolated CD8 ${ }^{+} \mathrm{T}$ lymphocytes were stimulated overnight with plate-bound anti-CD3 in the presence or absence of sULBP4 before collecting supernatants for ELISA measurements. (D) Quantification of secreted GM-CSF (D.a) and IFNy (D.b) levels (pg/mL) in the presence (blue bars) or absence (white bars) of rhULBP4. Each dot represents 1 donor. Mean, $n=10$ per group. Paired $t$ test for GM-CSF production and Wilcoxon test for IFNy production comparing NIL vS SULBP4; ${ }^{*} p<0.05$ and ** $p<0.01$. GM-CSF = granulocyte-macrophage colony-stimulating factor; MS = multiple sclerosis; rhULBP4 = recombinant human ULBP4; sULBP4 = soluble UL16-binding protein 4; ULBP4 = UL16-binding protein 4. 
Soluble ULBP4 Is Present in the CSF and Can Boost the Inflammatory Properties of CD8 ${ }^{+}$ T Lymphocytes.

We have previously established that murine UL16-binding proteinlike transcript 1 (MULT1) is the most abundantly expressed murine NKG2DL in the $\mathrm{MOG}_{35-55}$ EAE mouse model ${ }^{8}$ and that a shed/cleaved form of MULT1 is enriched in the CSF of EAE mice. ${ }^{8}$ Therefore, we tested whether the most abundant NKG2DL detectable in MS brain samples, ULBP4, is also enriched in the CSF of patients with MS. The presence of ULBP4 in CSF from patients with MS and controls was assessed by Western blot (eMethods, links.lww.com/NXI/A669). We detected 2 sets of bands for ULBP4 (Figure 4A): 1 that migrated at $45-50 \mathrm{kDa}$ and thus corresponded to the full size protein, similar to what we detected in brain lysates (Figure 1B). Additional bands, migrating around $25 \mathrm{kDa}$, were also observed, possibly corresponding to shed forms of the extracellular part of ULBP4. Although we observed comparable levels of the $45-50-\mathrm{kDa}$ ULBP4 in CSF from MS and controls, the $25 \mathrm{kDa}$ form of ULBP4 was elevated in the CSF of female patients with MS (Figure 4B) compared with male patients with MS and controls of both sexes.
The soluble form of MULT1, an elevated murine NKG2DL in the CSF of EAE mice, ${ }^{8}$ can boost effector functions of murine NK cells ${ }^{18}$ and $\mathrm{CD} 8^{+} \mathrm{T}$ lymphocytes. ${ }^{8}$ Notably, human $\mathrm{CD}^{+} \mathrm{T}$ lymphocytes are a predominant NKG2Dexpressing cell type in MS lesions. ${ }^{7}$ Therefore, we investigated whether soluble ULBP4, which is elevated in the CSF from female patients with MS, has an impact on $\mathrm{CD} 8^{+}$ $\mathrm{T}$ lymphocyte functions. The addition of soluble ULBP4 to human $\mathrm{CD}^{+} \mathrm{T}$ lymphocytes activated with anti-CD3 (Figure 4C) significantly increased the secretion of granulocyte-macrophage colony-stimulating factor (GM-CSF) and IFN $\gamma$ (Figure 4, C and D), 2 key inflammatory cytokines. The data suggest that a soluble form of ULBP4, which is enriched in the CSF of female patients with MS, can enhance the production of GM-CSF and IFN $\gamma$ by activated human $\mathrm{CD}^{+} \mathrm{T}$ lymphocytes.

\section{Soluble ULBP4 Increases CD ${ }^{+}$T Lymphocyte Motility and Enhances Kinapse-Like Behaviors} To evaluate the impact of soluble ULBP4 on human CD8 ${ }^{+}$ $\mathrm{T}$ lymphocytes entering the CNS, we used our established live imaging cocultured human astrocyte- $\mathrm{CD}^{+} \mathrm{T}$ lymphocyte

Figure 5 Soluble ULBP4 Enhances the Motility of CD8 ${ }^{+}$T Lymphocytes
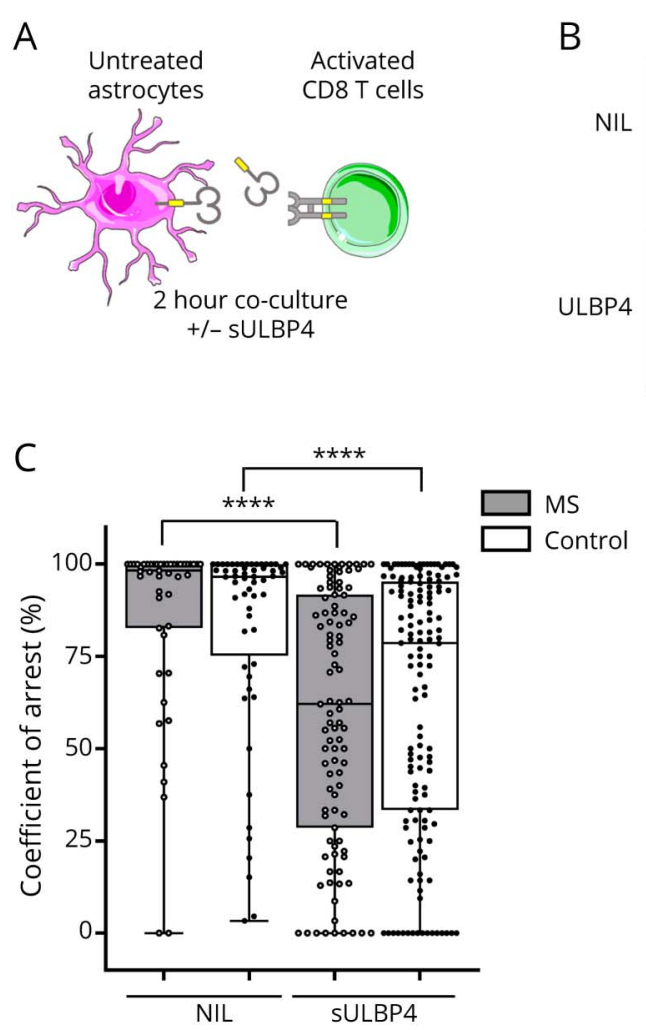

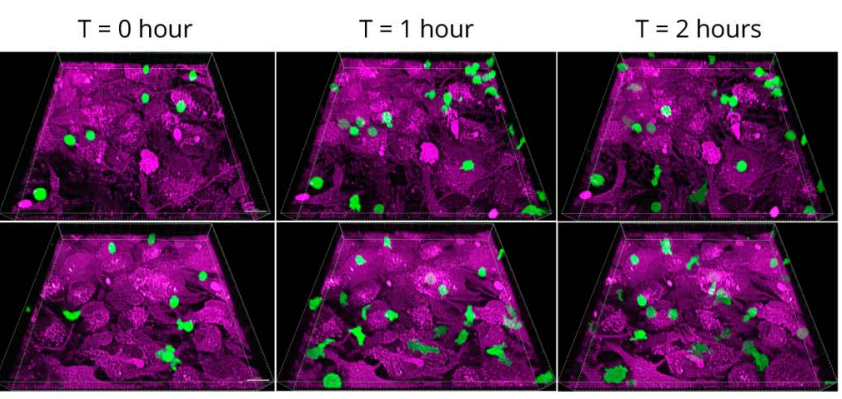

D

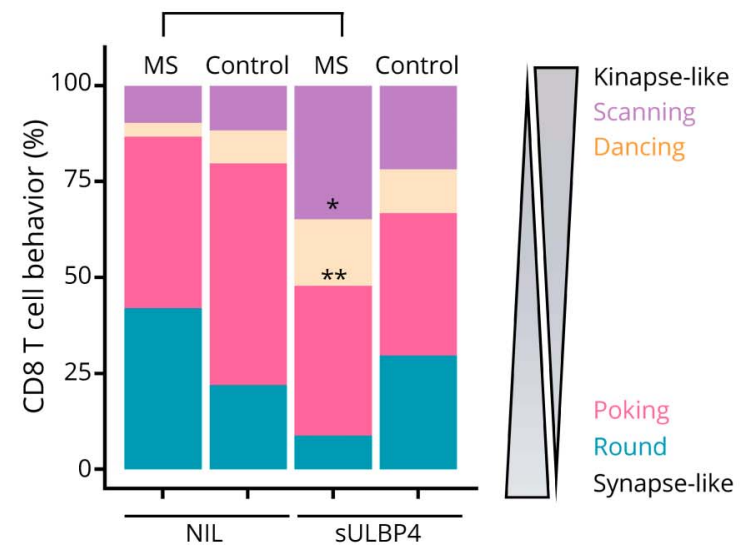

(A-D) Time-lapse imaging of activated $C D 8^{+} \mathrm{T}$ lymphocytes cocultured with human astrocytes in the presence or absence of sULBP4. (A) Diagram illustrating culture conditions. CD8 ${ }^{+}$T lymphocytes were activated overnight on anti-CD3 coated plates in the presence of anti-CD28 and interleukin-15, CFSE-labeled, and then added to astrocytes in the presence or absence of sULBP4 and imaged for 2 hours. (B) Three-dimensional time-lapse view at T = 0 , $T=1$, and T = 2 hours of activated $\mathrm{CD}^{+} \mathrm{T}$ lymphocytes (green) cocultured with astrocytes (magenta). (C and D) Analysis of the motility and behavior of CD8 ${ }^{+} \mathrm{T}$ lymphocytes from 3 untreated patients with MS and 3 sex- and age-matched healthy controls on coculture with astrocytes in the presence or absence of sUBLP4. Presented data are pooled from 3 independent experiments. (C) Coefficient of arrest (\%) of individual CD8 ${ }^{+} \mathrm{T}$ lymphocytes; each dot represents 1 cell. Kruskal-Wallis test comparing sULBP4 vs NIL for each donors' group $* \star \star \star x p<0.0001$. (D) Proportion of CD8 ${ }^{+} T$ lymphocytes exhibiting the scanning, dancing, poking, and round behaviors when cocultured with astrocytes in the presence or absence of sULBP4. One way analysis of variance comparing NIL vs sULBP4 for each donors' group, ${ }^{\star} p<0.05, \star \star *<$ 0.01. CFSE = carboxyfluorescein succinimidyl ester; MS = multiple sclerosis; sULBP4 = soluble UL16-binding protein 4; ULBP4 = UL16-binding protein 4. 
assay (Figure 5, A and B, Videos, eMethods, links.lww.com/NXI/ A669). We compared the motility and behavior of activated $\mathrm{CD} 8^{+}$ $\mathrm{T}$ lymphocytes from healthy donors and patients with MS. ${ }^{19}$ Adding soluble ULBP4 to astrocyte-CD8 T lymphocyte cocultures decreased the coefficient of arrest of $\mathrm{CD} 8^{+} \mathrm{T}$ lymphocytes, supporting an increased motility (Figure 5, A-C). Notably, this effect was more pronounced for $\mathrm{CD}^{+} \mathrm{T}$ lymphocytes from patients with MS compared with healthy controls.

We have previously identified in astrocyte-CD8 T-cell coculture 4 different $\mathrm{CD}^{+} \mathrm{T}$ lymphocyte behaviors: round, poking, dancing, and scanning. ${ }^{19}$ Poking and round behaviors are associated with synapse-like behaviors, which are characterized by long-lasting interactions between $\mathrm{T}$ lymphocytes and antigen-presenting cells and migration arrest of $\mathrm{T}$ lymphocytes. ${ }^{20-22}$ Scanning and dancing behaviors are associated with kinapse-like behaviors, which refer to more dynamic interactions between antigen-presenting cells and $\mathrm{T}$ lymphocytes and thus higher motility of T lymphocytes. ${ }^{19}$ The addition of soluble ULBP4 in the coculture induced a significant increase in the proportion of $\mathrm{CD}^{+} \mathrm{T}$ lymphocytes adopting a dancing or scanning behavior (Figure 5, B-D). Although we could observe these changes in $\mathrm{CD} 8^{+}$ $\mathrm{T}$ lymphocytes from both patients with MS and controls, these differences reached significance for MS T lymphocytes, indicating that these cells were more sensitive to soluble ULBP4. These behavioral changes suggest that $\mathrm{CD}^{+}$ $\mathrm{T}$ lymphocytes from patients with MS respond to the soluble form of ULBP4 detected in CSF by increasing their motility and adopting kinapse-like behaviors.

\section{Discussion}

Several cellular stressors present in chronic inflammatory and autoimmune diseases have been shown to upregulate the expression of specific NKG2DLs, consequently leading to the activation of NKG2D-bearing immune effector cells. ${ }^{5}$ In this study, we provide new evidence for the involvement of 1 specific NKG2DL, ULBP4, in the pathobiology of MS, a prototypic neuroinflammatory disease (Figure 6). We demonstrate elevated ULBP4 protein expression in postmortem brain tissues from patients with MS (Figure 1) and identify astrocytes as the predominant cells expressing this ligand (Figure 2). In addition, our data demonstrate that stressors present in MS lesions can trigger ULBP4 expression by human astrocytes (Figure 3). We also demonstrate elevated levels of a shed/soluble form of ULBP4 in CSF from female patients with MS compared with male patients with MS and female and male controls (Figure 4). Finally, we establish a novel role for soluble ULBP4 in enhancing effector properties (Figure 4) and motility of human $\mathrm{CD} 8^{+} \mathrm{T}$ lymphocytes, with a stronger impact on cells from patients with MS (Figure 5).

NKG2DLs alert the immune system when tissue homeostasis is compromised in various contexts such as tumor, infection,

Figure 6 Proposed Involvement of the NKG2D Pathway in MS Pathology

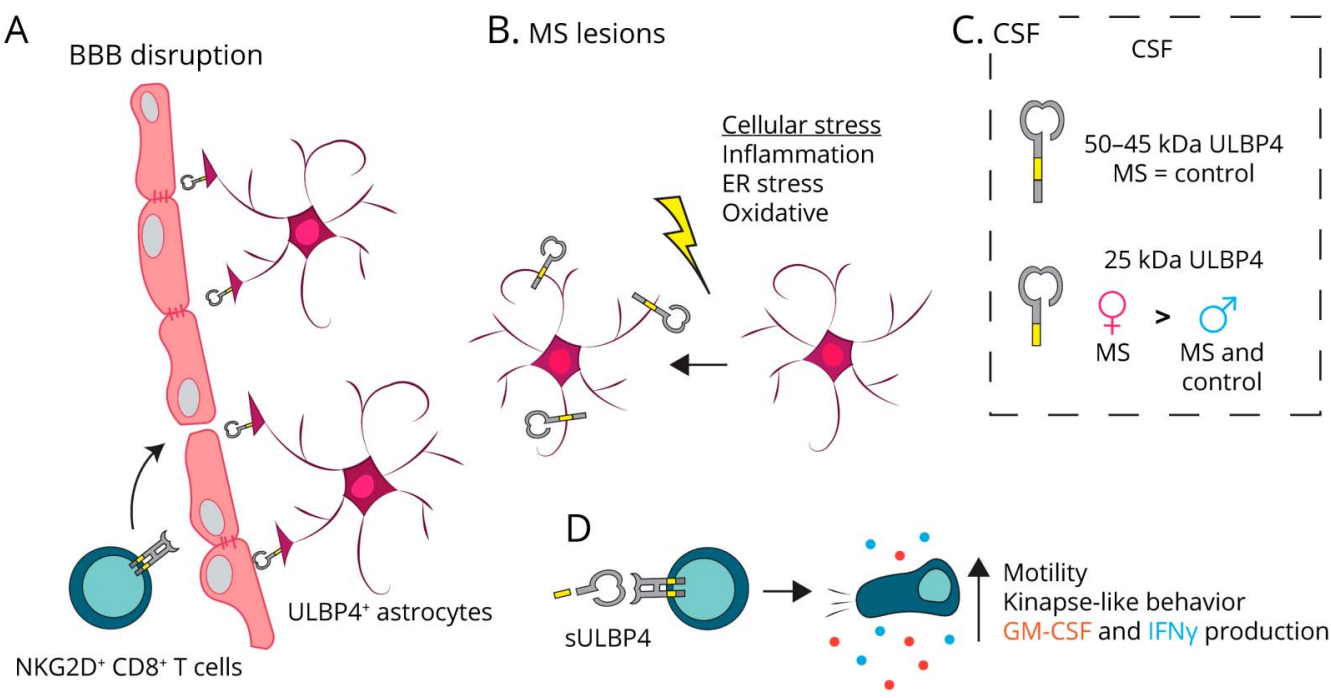

(A) CD8 ${ }^{+}$T lymphocytes can enter the CNS of patients with MS in part due to disrupted BBB. All infiltrating CD8 ${ }^{+}$T lymphocytes express NKG2D ${ }^{7}$ and thus can interact with ULBP4-expressing astrocytes including those having end feet in close proximity to the BBB. (B) Various cellular stresses (inflammation, ER stress, and oxidative stress) present in the brain of patients with MS can upregulate ULBP4 expression by astrocytes, as supported by our in vitro data. Reactive astrocytes, which are abundantly present in MS lesion, represent the predominant cell type expressing ULBP4 in the brain of patients with MS. (C) Soluble forms of ULBP4 are detectable in CSF; a soluble 25-kDa ULBP4 form is significantly elevated in CSF from female patients with MS compared with groups (male patients with MS and controls of both sexes). (D) Soluble ULBP4 can affect immune cell functions. On contact with sULBP4, CD8 ${ }^{+}$T lymphocytes increase their secretion of GM-CSF and IFNy. Moreover, addition of SULBP4 to CD8 ${ }^{+}$T lymphocytes cocultured with astrocytes increases their motility and favors kinapse-like behaviors, which are more dynamic and may lead to enhanced displacement within the tissue. BBB = blood-brain barrier; ER = endoplasmic reticulum; MS = multiple sclerosis; sULBP4 = soluble UL16-binding protein 4. 
inflammation, and cellular stress. Because immune effector cells express the NKG2D receptor under normal physiologic conditions, the tight regulation of ligand expression is key to control the activation of the NKG2D-NKG2DL pathway. ${ }^{23}$ Indeed, NKG2DLs are subjected to transcriptional, posttranscriptional, translational, and posttranslational regulatory mechanisms that modulate their expression. ${ }^{10,11}$ We detected mRNA coding for the 8 identified human NKG2DLs (ULBP1-6 and MICA, B) in human brain tissues from both patients with MS and controls (Figure 1 and eFigure 1, links. lww.com/NXI/A669). However, ULBP4 was the only ligand we could detect by Western blot in brain lysates (Figure 1 and eFigure 1) using commercially available antibodies ( $R \& D$ Systems) widely used by the scientific community. Bands of different molecular weights were detected for ULBP4 by Western blot (Figure 1). We speculate that these differences in size are likely due to posttranslational modifications such as glycosylation, which has been reported for ULBP4. ${ }^{24}$ We have previously reported the detection of MICA/B in a limited number of snap-frozen postmortem MS brain tissues $(\mathrm{n}=2)$ and colocalization with $\mathrm{O} 4$ (oligodendrocyte marker) but not GFAP. $^{14}$ It is possible that other NKG2DLs, including $\mathrm{MICA} / \mathrm{B}$, are present in MS brain tissues but that these ligands do not reach levels comparable to ULBP4. We used the same amount of protein (50 ng) of the positive controls, which are recombinant NKG2DLs obtained from the same vendor, to confirm our capacity to detect NKG2DLs by Western blot (eFigure 1). However, no signal could be detected in the CNS samples for non-ULBP4 ligands. ULBP4 protein expression was frequently observed in astrocytes surrounding blood vessels (Figure 2), suggesting that incoming NKG2D ${ }^{+}$immune cells, which include all infiltrating $\mathrm{CD}^{+} \mathrm{T}$ lymphocytes and a subset of $\mathrm{CD} 4^{+} \mathrm{T}$ lymphocytes, ${ }^{7}$ could encounter ULBP4-expressing astrocytes. Notably, higher NKG2DL expression in the gut of patients with $\mathrm{Crohn}^{25}$ and celiac diseases ${ }^{26}$ compared with healthy donors supports the notion that pathologically elevated NKG2DL levels can contribute to chronic inflammatory and autoimmune diseases.

Increasing evidence supports a key role for astrocytes in MS pathobiology throughout different disease stages. ${ }^{3}$ In MS brains, these abundant glial cells are subjected to inflammatory mediators, which can induce glial reactivity, but also lead to ER stress and oxidative damage. ${ }^{1}$ Astrocytes upregulate ER stress-related proteins, including ViP and the C/EBP homologous protein, in MS lesions as well as NAWM.$^{27}$ Notably, significantly higher levels of ULBP4 were detected in MS lesions and NAWM (Figures 1, 2, and 6). Our in vitro data validate that indeed inflammation, ER stress, and oxidative stress increased the proportion of human astrocytes expressing ULBP4 (Figures 3 and 6) but did not alter expression in human neurons (eFigure 2, links.lww.com/NXI/ A669), suggesting a specific response of astrocytes to these stressors. Others have shown NKG2D-mediated killing of human astrocytes by activated NK cells in a mixed neuronastrocyte culture, supporting the preferential expression of
NKG2DLs by astrocytes. ${ }^{28}$ Our results demonstrating differential responses to cellular stress between human neurons and astrocytes are not surprising as numerous publications document that distinct cell types upregulate NKG2DLs in response to various stresses, including the MS-relevant stressors used here. ${ }^{11,29,30}$ Finally, as cellular stress such as inflammation, ER stress, and oxidative stress are observed in other neurologic disorders, ${ }^{31,32}$ it will be relevant to investigate whether NKG2DLs are also upregulated in these conditions.

Soluble forms of NKG2DLs have been observed in several conditions; a variety of mechanisms have been implicated in the release of these soluble ligands, and their impact on immune functions varies depending on the specific ligand, cell type, and context. ${ }^{33}$ Several groups have shown that in cancer, some tumor-derived soluble NKG2DLs are associated with poor prognosis and can downmodulate NKG2D and restrain NK and T-cell cytotoxicity. ${ }^{34,35}$ In contrast, soluble MULT1, a murine NKG2DL, can enhance NK cell activity and promote tumor rejection. ${ }^{18}$ Likewise, we have shown that soluble MULT1 is elevated in the CSF of EAE mice and can increase the inflammatory properties of $\mathrm{CD} 8^{+}$ T lymphocytes. ${ }^{8}$ We now show that a soluble form of ULBP4 $(25 \mathrm{kDa})$ is elevated in the CSF of female patients with MS compared with male patients with MS and controls (Figures 4 and 6). Despite this difference, CSF from all male and female patients with MS was positive for oligoclonal bands, and both patient groups included similar proportions of patients with PPMS and RRMS. We speculate that sexspecific inflammatory processes in the CNS of female patients could lead to the release of this soluble form of ULBP4. Notably, others compared postmortem tissue from female and male patients with MS and observed multiple differences in mRNA levels of genes coding for hormone receptors and cytokines. ${ }^{36}$ Moreover, large transcriptomic analyses performed in animal models have documented that glial cells, including astrocytes, exhibit sex differences in their transcriptomic patterns. ${ }^{37,38}$ Additional tools will be necessary to identify the mechanisms driving this sex difference. Moreover, splice variants of ULBP4 have been reported in immortalized tumor cell lines including soluble forms of various sizes. ${ }^{24,39,40}$ However, whether these variants can be expressed by nonimmortalized cells or influenced by sex has not been shown. Unfortunately, a lack of available tools to distinguish different ULBP4 isoforms prevents us from further investigating the $25 \mathrm{kDa}$ soluble form.

We show that soluble ULBP4, similarly to MULT1 in mice, boosts the production of 2 key inflammatory cytokines, GM-CSF and IFN $\gamma$, by activated human $\mathrm{CD} 8^{+}$ $\mathrm{T}$ lymphocytes (Figures 4 and 6). We demonstrate that soluble ULBP4 significantly modifies the behavior of activated human $\mathrm{CD}^{+} \mathrm{T}$ lymphocytes encountering human astrocytes, especially $\mathrm{T}$ lymphocytes from patients with MS (Figures 5 and 6). Indeed, the addition of soluble ULBP4 favored kinapse-like behavior by $\mathrm{CD} 8^{+} \mathrm{T}$ lymphocytes while 
reducing the proportion of $\mathrm{T}$ cells involved in synapse-like behaviors (Figures 5 and 6). These changes cannot be explained by altered NKG2D expression, as both patients with MS and controls showed similar NKG2D levels, and the presence of soluble ULBP4 did not downregulate the receptor (data not shown). We ruled out that ULBP4 has chemotactic properties, which could increase T lymphocyte motility; indeed, adding soluble ULBP4 in a transwell chemotaxis assay had no impact on $\mathrm{CD}^{+} \mathrm{T}$ lymphocyte migration (data not shown). We speculate that the presence of soluble ULBP4 enhances movement of $\mathrm{T}$ lymphocytes within the brain parenchyma via nonchemoattractive mechanisms.

A single injection of an $\alpha$-NKG2D neutralizing antibody reduced disease activity in patients with Crohn disease ${ }^{41}$ supporting the feasibility of targeting the NKG2D pathway in inflammatory diseases in humans. However, as the NKG2D pathway contributes to the immunosurveillance of infected cells and tumors, it is imperative to determine whether there is cell type specificity that can be exploited to refine NKG2Dtargeted therapeutic approaches. Indeed, targeting ULBP4, without altering other ligands that might contribute to important immune functions, could yield a very specific therapy. Importantly, it is known that ULBP4 is implicated in various types of cancers ${ }^{42-44}$; however, current data suggest that ULBP4 is not expressed in CNS-related cancers in adults. Although MICA, MICB, and ULBP1-2 are detected at the protein level in human adult glioblastoma multiforme (GBM) and meningioma samples, ULBP3 and ULBP4 are not. ${ }^{45,46}$ In addition, MICB and ULBP1 are detected on GBM-infiltrating myeloid cells. ${ }^{46}$ In pediatric cases of low grade glioma, elevated levels of ULBP2/5/6 and ULBP4 have been observed, although not in high-grade glioma samples. ${ }^{47}$ Additional studies will be required to fully characterize the expression pattern of ULBP4 in the human brain; nevertheless, available data including our current study suggest that in adults, ULBP4 is not associated with brain tumors but rather is increased in patients with MS. Therefore, our study suggests that ULBP4 could be a valid target in MS pathobiology without impairing beneficial immunosurveillance in the CNS (Figure 6).

\section{Acknowledgment}

The authors are grateful to the team of Dr. Elie Haddad at the CHU-Ste-Justine and the Birth Defects Research Laboratory (BDRL) of Dr. Ian Glass at the University of Washington for providing fetal CNS tissues. They also thank the cellular imaging core facility and the flow cytometry core facility of the CRCHUM as well as Sandra Larouche for her help with cryosectioning brain tissues.

\section{Study Funding}

This study was supported by an Operating Grant from the Canadian Institutes of Health Research held by N.A. (CIHR, PTJ-168870). A.C.M. and S.E.J.Z., respectively, obtained a joint studentship and postdoctoral award from the FRQS-MSSC. N.F-k. obtained studentships from the Neuroinflammation-
CIHR training program and FRQS. C.L. holds a ChercheurBoursier Junior 1 salary award from FRQS, and A.P. holds a Canadian Research Chair Tier I in Multiple Sclerosis. The CIHR had no role in the design of this study, its execution, and the analyses and interpretation of the data.

\section{Disclosure}

The authors report no disclosures relevant to the manuscript. Go to Neurology.org/NN for full disclosures.

\section{Publication History}

Received by Neurology: Neuroimmunology \& Neuroinflammation August 17, 2021. Accepted in final form October 19, 2021.

Appendix Authors

\begin{tabular}{|c|c|c|}
\hline Name & Location & Contribution \\
\hline $\begin{array}{l}\text { Ana } \\
\text { Carmena } \\
\text { Moratalla, } \\
\text { MSc }\end{array}$ & $\begin{array}{l}\text { Department of Neuroscience, } \\
\text { Faculty of Medicine, Université } \\
\text { de Montréal } \\
\text { Centre de recherche du Centre } \\
\text { Hospitalier de l'Université de } \\
\text { Montréal (CRCHUM) Montréal, } \\
\text { Canada }\end{array}$ & $\begin{array}{l}\text { Conducted experiments, } \\
\text { analyzed and interpreted } \\
\text { data, and wrote the } \\
\text { manuscript }\end{array}$ \\
\hline
\end{tabular}

\begin{tabular}{lll}
\hline $\begin{array}{l}\text { Yves } \\
\text { Carpentier } \\
\text { Solorio, BSc }\end{array}$ & $\begin{array}{l}\text { Department of Neuroscience, } \\
\text { Faculty of Medicine, Université } \\
\text { de Montréal } \\
\text { Centre de recherche du Centre } \\
\text { Hospitalier de l'Université de } \\
\text { Montréal (CRCHUM) Montréal, } \\
\text { Canada }\end{array}$ & $\begin{array}{l}\text { Conducted experiments, } \\
\text { analyzed and interpreted } \\
\text { data, and revised the } \\
\text { manuscript }\end{array}$ \\
\hline $\begin{array}{l}\text { Florent } \\
\text { Lemaitre, } \\
\text { MSc }\end{array}$ & $\begin{array}{l}\text { Department of Neuroscience, } \\
\text { Faculty of Medicine, Université } \\
\text { de Montréal } \\
\text { Centre de recherche du Centre }\end{array}$ & $\begin{array}{l}\text { Conducted experiments } \\
\text { and revised the } \\
\text { manuscript }\end{array}$ \\
& $\begin{array}{l}\text { Hospitalier de l'Université de } \\
\text { Montréal (CRCHUM) Montréal, } \\
\text { Canada }\end{array}$
\end{tabular}

\begin{tabular}{lll}
\hline Negar & Department of Neuroscience, & Conducted experiments, \\
Farzam-kia, & Faculty of Medicine, Université \\
BSc & $\begin{array}{l}\text { de Montréal } \\
\text { and revised the } \\
\text { manuscript }\end{array}$ \\
& $\begin{array}{l}\text { Centre de recherche du Centre } \\
\text { Hospitalier de I'Université de }\end{array}$ \\
& Montréal (CRCHUM) Montréal, \\
& Canada
\end{tabular}

\begin{tabular}{|c|c|c|}
\hline $\begin{array}{l}\text { Annie } \\
\text { Levert, DEC }\end{array}$ & $\begin{array}{l}\text { Centre de recherche du Centre } \\
\text { Hospitalier de l'Université de } \\
\text { Montréal (CRCHUM) Montréal, } \\
\text { Canada }\end{array}$ & Conducted experiments \\
\hline $\begin{array}{l}\text { Stephanie } \\
\text { E.J. Zandee, } \\
\text { PhD }\end{array}$ & $\begin{array}{l}\text { Department of Neuroscience, } \\
\text { Faculty of Medicine, Université } \\
\text { de Montréal } \\
\text { Centre de recherche du Centre } \\
\text { Hospitalier de I'Université de } \\
\text { Montréal (CRCHUM) Montréal, } \\
\text { Canada }\end{array}$ & $\begin{array}{l}\text { Was involved in the } \\
\text { collection of human } \\
\text { samples, provided } \\
\text { important scientific input, } \\
\text { and revised the } \\
\text { manuscript }\end{array}$ \\
\hline
\end{tabular}

Boaz Lahav, Multiple Sclerosis CHUM Clinic Was involved in the BSC Centre de recherche du Centre collection of human Hospitalier de l'Université de samples and clinical Montréal (CRCHUM) Montréal, characterization of Canada patients

\begin{tabular}{lll}
\hline Jean Victor & CLSC des Faubourgs. CIUSSS & Was involved in the \\
Guimond, & du Centre-Sud-de-l'lle-de- & collection of human \\
MD & $\begin{array}{l}\text { Montréal, Montréal, QC, } \\
\text { Canada }\end{array}$ & samples \\
&
\end{tabular}


Appendix (continued)

\begin{tabular}{lll}
\hline Name & Location & Contribution \\
\hline $\begin{array}{ll}\text { Elie Haddad, } \\
\text { MD, PhD }\end{array}$ & $\begin{array}{l}\text { Department of Microbiology, } \\
\text { Infectious Diseases, and }\end{array}$ & $\begin{array}{l}\text { Was involved in the } \\
\text { collection of human } \\
\text { Immunology and Department } \\
\text { samples } \\
\text { of Pediatrics, Université de }\end{array}$ \\
& $\begin{array}{l}\text { Montréal, Centre de Recherche } \\
\text { du Centre Hospitalier }\end{array}$ \\
& Universitaire Sainte-Justine \\
& (CHU Sainte-Justine), Montreal, \\
& QC, Canada.
\end{tabular}

\begin{tabular}{|c|c|c|}
\hline $\begin{array}{l}\text { Marc Girard, } \\
\text { MD }\end{array}$ & $\begin{array}{l}\text { Multiple Sclerosis CHUM Clinic } \\
\text { Department of Neuroscience, } \\
\text { Faculty of Medicine, Université } \\
\text { de Montréal } \\
\text { Centre de recherche du Centre } \\
\text { Hospitalier de l'Université de } \\
\text { Montréal (CRCHUM) Montréal, } \\
\text { Canada }\end{array}$ & $\begin{array}{l}\text { Was involved in the } \\
\text { collection of human } \\
\text { samples and clinica } \\
\text { characterization of } \\
\text { patients }\end{array}$ \\
\hline
\end{tabular}

\begin{tabular}{lll}
\hline Pierre & Multiple Sclerosis CHUM Clinic & Was involved in the \\
Duquette, & Department of Neuroscience, & collection of human \\
MD & Faculty of Medicine, Université & samples and clinical \\
& de Montréal & characterization of \\
& Centre de recherche du Centre & patients and revised the \\
& Hospitalier de l'Université de & manuscript. \\
& Montréal (CRCHUM) Montréal, \\
& Canada
\end{tabular}

\begin{tabular}{lll}
\hline Catherine & Multiple Sclerosis CHUM Clinic & Was involved in the \\
Larochelle, & Department of Neuroscience, & collection of human \\
MD, PhD & Faculty of Medicine, Université & samples and clinical \\
& de Montréal & characterization of \\
& Centre de recherche du Centre & patients and revised the \\
& Hospitalier de l'Université de & manuscript. \\
& Montréal (CRCHUM) Montréal, \\
& Canada
\end{tabular}

\begin{tabular}{lll}
\hline $\begin{array}{l}\text { Alexandre } \\
\text { Prat, MD, }\end{array}$ & Multiple Sclerosis CHUM Clinic & Was involved in the \\
PhD & Department of Neuroscience, & collection of human \\
& de Montréal & samples and clinical \\
& characterization of \\
& Centre de recherche du Centre & patients. \\
& Hospitalier de l'Université de & \\
& Montréal (CRCHUM) Montréal, \\
& Canada
\end{tabular}

Nathalie Department of Neuroscience, Designed the study,

Arbour, PhD Faculty of Medicine, Université analyzed and interpreted de Montréal the data, wrote the Centre de recherche du Centre manuscript, and secured Hospitalier de l'Université de funding. Montréal (CRCHUM) Montréal, Canada
9. Babic M, Dimitropoulos C, Hammer Q et al. NK cell receptor NKG2D enforces proinflammatory features and pathogenicity of Th1 and Th17 cells. J Exp Med. 2020; 217(8):e20190133.

10. Zingoni A, Molfetta R, Fionda C, et al. NKG2D and its ligands: "one for all, all for one". Front Immunol. 2018;9:476.

11. Raulet DH, Gasser S, Gowen BG, Deng W, Jung H. Regulation of ligands for the NKG2D activating receptor. Annu Rev Immunol. 2013;31:413-441.

12. Groh V, Bruhl A, El-Gabalawy H, Nelson JL, Spies T. Stimulation of T cell autoreactivity by anomalous expression of NKG2D and its MIC ligands in rheumatoid arthritis. Proc Natl Acad Sci USA. 2003;100(16):9452-9457.

13. Fernandez-Morera JL, Rodriguez-Rodero S, Lahoz C, et al. Soluble MHC class I chain-related protein B serum levels correlate with disease activity in relapsingremitting multiple sclerosis. Hum Immunol. 2008;69(4-5):235-240.

14. Saikali P, Antel JP, Newcombe J, et al. NKG2D-mediated cytotoxicity toward oligodendrocytes suggests a mechanism for tissue injury in multiple sclerosis. J Neurosci. 2007;27(5):1220-1228.

15. Thompson AJ, Banwell BL, Barkhof F, et al. Diagnosis of multiple sclerosis: 2017 revisions of the McDonald criteria. Lancet Neurol. 2018;17(2):162-173.

16. Stone $\mathrm{S}, \mathrm{Lin} \mathrm{W}$. The unfolded protein response in multiple sclerosis. Front Neurosci. 2015;9:264

17. Göbel K, Ruck T, Meuth SG. Cytokine signaling in multiple sclerosis: lost in translation. Mult Scler. 2018;24(4):432-439.

18. Deng W, Gowen BG, Zhang L, et al. Antitumor immunity. A shed NKG2D ligand that promotes natural killer cell activation and tumor rejection. Science. 2015;348(6230):136-139.

19. Lemaître F, Carmena Moratalla A, Farzam-Kia N, et al. Capturing T lymphocytes' dynamic interactions with human neural cells using time-lapse microscopy. Front Immunol. 2021;12:668483.

20. Dustin ML. Cell adhesion molecules and actin cytoskeleton at immune synapses and kinapses. Curr Opin Cell Biol. 2007;19(5):529-533.

21. Moreau HD, Lemaitre F, Garrod KR, Garcia Z, Lennon-Duménil AM, Bousso P. Signal strength regulates antigen-mediated T-cell deceleration by distinct mechanisms to promote local exploration or arrest. Proc Natl Acad Sci USA. 2015;112(39): 12151-12156.

22. Friedman RS, Beemiller P, Sorensen CM, Jacobelli J, Krummel MF. Real-time analysis of $\mathrm{T}$ cell receptors in naive cells in vitro and in vivo reveals flexibility in synapse and signaling dynamics. J Exp Med. 2010;207(12):2733-2749.

23. Stern-Ginossar N, Mandelboim O. An integrated view of the regulation of NKG2D ligands. Immunology. 2009;128:1-6.

24. Zöller T, Wittenbrink M, Hoffmeister M, Steinle A. Cutting an NKG2D ligand short: cellular processing of the peculiar human NKG2D ligand ULBP4. Front Immunol. 2018;9:620

25. Allez M, Tieng V, Nakazawa A, et al. CD4+NKG2D+ T cells in Crohn's disease mediate inflammatory and cytotoxic responses through MICA interactions. Gastroenterology. 2007;132(7):2346-2358.

26. Hüe S, Mention JJ, Monteiro RC, et al. A direct role for NKG2D/MICA interaction in villous atrophy during celiac disease. Immunity. 2004;21(3):367-377.

27. Cunnea P, Mháille AN, McQuaid S, Farrell M, McMahon J, FitzGerald U. Expression profiles of endoplasmic reticulum stress-related molecules in demyelinating lesions and multiple sclerosis. Mult Scler. 2011;17(7):808-818.

28. Darlington PJ, Podjaski C, Horn KE, et al. Innate immune-mediated neuronal injury consequent to loss of astrocytes. J Neuropathol Exp Neurol. 2008;67(6): 590-599.

29. Stojanovic A, Correia MP, Cerwenka A. The NKG2D/NKG2DL Axis in the crosstalk between lymphoid and myeloid cells in health and disease. Front Immunol. 2018;9; 827.

30. Hosomi S, Grootjans J, Huang YH, Kaser A, Blumberg RS. New insights into the regulation of natural-killer group 2 member D (NKG2D) and NKG2D-ligands: endoplasmic reticulum stress and CEA-related cell adhesion molecule 1. Front Immunol. 2018;9:1324.

31. Sims SG, Cisney RN, Lipscomb MM, Meares GP. The role of endoplasmic reticulum stress in astrocytes. Glia. 2021;70(1):5-19.

32. Quincozes-Santos A, Santos CL, de Souza Almeida RR, et al. Gliotoxicity and glioprotection: the dual role of glial cells. Mol Neurobiol. 2021.

33. Molfetta R, Quatrini L, Santoni A, Paolini R. Regulation of NKG2D-dependent NK cell functions: the yin and the yang of receptor endocytosis. Int J Mol Sci. 2017;18(8): 1677.

34. Luo Q, Luo W, Zhu Q, et al. Tumor-derived soluble MICA obstructs the NKG2D pathway to restrain NK cytotoxicity. Aging Dis. 2020;11(1):118-128.

35. Groh V, Wu J, Yee C, Spies T. Tumour-derived soluble MIC ligands impair expression of NKG2D and T-cell activation. Nature. 2002;419(6908):734-738.

36. Luchetti S, van Eden CG, Schuurman K, van Strien ME, Swaab DF, Huitinga I. Gender differences in multiple sclerosis: induction of estrogen signaling in male and progesterone signaling in female lesions. J Neuropathol Exp Neurol. 2014;73(2): 123-135.

37. Lu T, Mar JC. Investigating transcriptome-wide sex dimorphism by multi-level analysis of single-cell RNA sequencing data in ten mouse cell types. Biol Sex Differ. 2020;11(1):61.

38. Mizrak D, Levitin HM, Delgado AC, et al. Single-cell analysis of regional differences in adult V-SVZ neural stem cell lineages. Cell Rep. 2019;26(2):394-406.e5.

39. Cao W, Xi X, Hao Z, et al. RAET1E2, a soluble isoform of the UL16-binding protein RAET1E produced by tumor cells, inhibits NKG2D-mediated NK cytotoxicity. J Biol Chem. 2007;282(26):18922-18928. 
40. Cao W, Xi X, Wang Z, et al. Four novel ULBP splice variants are ligands for human NKG2D. Int Immunol. 2008;20(8):981-991.

41. Allez M, Skolnick BE, Wisniewska-Jarosinska M, Petryka R, Overgaard RV. AntiNKG2D monoclonal antibody (NNC0142-0002) in active Crohn's disease: a randomised controlled trial. Gut. 2017;66(11):1918-1925.

42. McGilvray RW, Eagle RA, Rolland P, Jafferji I, Trowsdale J, Durrant LG. ULBP2 and RAET1E NKG2D ligands are independent predictors of poor prognosis in ovarian cancer patients. Int J Cancer. 2010;127(6):1412-1420.

43. Fujita $H$, Hatanaka $Y$, Sutoh $Y$, et al. Immunohistochemical validation and expression profiling of NKG2D ligands in a wide spectrum of human epithelial neoplasms. J Histochem Cytochem. 2015;63(3):217-227.
44. Okita R, Maeda A, Shimizu K, Nojima Y, Saisho S, Nakata M. Clinicopathological relevance of tumor expression of NK group 2 member $\mathrm{D}$ ligands in resected non-small cell lung cancer. Oncotarget. 2019;10(63):6805-6815.

45. Crane CA, Han SJ, Barry JJ, Ahn BJ, Lanier LL, Parsa AT. TGF-beta downregulates the activating receptor NKG2D on NK cells and CD8+ T cells in glioma patients. Neuro Oncol. 2010;12(1):7-13

46. Crane CA, Austgen $\mathrm{K}$, Haberthur $\mathrm{K}$, et al. Immune evasion mediated by tumorderived lactate dehydrogenase induction of NKG2D ligands on myeloid cells in glioblastoma patients. Proc Natl Acad Sci USA. 2014;111(35):12823-12828.

47. Haberthur K, Brennan K, Hoglund V, et al. NKG2D ligand expression in pediatric brain tumors. Cancer Biol Ther. 2016;17(12):1253-1265. 


\section{Neurology \\ Neuroimmunology \& Neuroinflammation}

\section{Stress Signal ULBP4, an NKG2D Ligand, Is Upregulated in Multiple Sclerosis and Shapes CD8 ${ }^{+}$T-Cell Behaviors Ana Carmena Moratalla, Yves Carpentier Solorio, Florent Lemaitre, et al. \\ Neurol Neuroimmunol Neuroinflamm 2022;9; DOI 10.1212/NXI.0000000000001119}

This information is current as of December 6, 2021

Updated Information \& Services

References

Subspecialty Collections

Permissions \& Licensing

Reprints including high resolution figures, can be found at: http://nn.neurology.org/content/9/1/e1119.full.html

This article cites 46 articles, 8 of which you can access for free at: http://nn.neurology.org/content/9/1/e1119.full.html\#\#ref-list-1

This article, along with others on similar topics, appears in the following collection(s):

All Immunology

http://nn.neurology.org//cgi/collection/all_immunology

Autoimmune diseases

http://nn.neurology.org//cgi/collection/autoimmune_diseases

Multiple sclerosis

http://nn.neurology.org//cgi/collection/multiple_sclerosis

Information about reproducing this article in parts (figures,tables) or in its entirety can be found online at:

http://nn.neurology.org/misc/about.xhtml\#permissions

Information about ordering reprints can be found online: http://nn.neurology.org/misc/addir.xhtml\#reprintsus

Neurol Neuroimmunol Neuroinflamm is an official journal of the American Academy of Neurology.

Published since April 2014, it is an open-access, online-only, continuous publication journal. Copyright

Copyright (C) 2021 The Author(s). Published by Wolters Kluwer Health, Inc. on behalf of the American

Academy of Neurology.. All rights reserved. Online ISSN: 2332-7812.

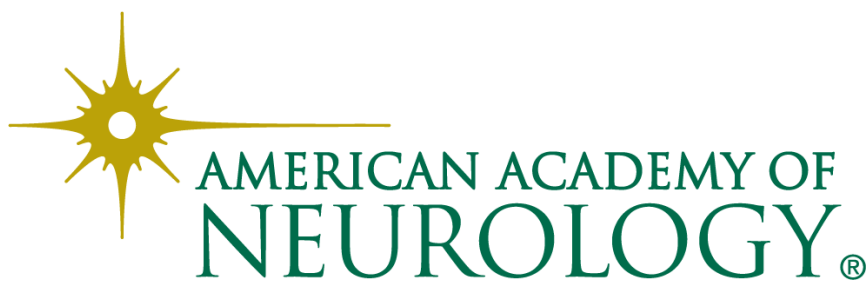

